\title{
Role of Ranibizumab in various retinal disorders
}

\author{
Dr.Pervez Ahemed Siddiqui, Dr.(Mrs.)Padmini Warkhede \\ ${ }^{1}$ Professor, M.B.B.S.M.S.(Ophthalmology),NSCB Medical College,Jabalpur(M.P.) \\ ${ }^{2}$ Asst.Professor ,M.B.B.S.,M.S.(Ophthlmology),N.S.C.B.Medical College,Jabalpur(M.P.)
}

\begin{abstract}
:
Purpose: Our aim was to evaluate role of anti VEGF Ranibizumab in diabetic CSME, Wet ARMD and retinal vein occlusion

Method: The present study was conducted in 100 patients who presented themselves in OPD of upgraded department of Ophthalmology,N.S.C.B.Medical college, Jabalpur (M.P.) during the academic session October 2014 - September 2016.All selected patients were given Intravitreal Ranibizumab on monthly basis after full history as well as thorough clinical examinations followed by treat and extend norms.OCT evaluation for central retinal thickness and visual acuity measurement was done prior to, after each injection as well as after completion of therapy.

Result: The mean no. of intravitreal Ranibizumab injections over 2 years was 5.6 per patient. Before starting therapy most of the patients had visual acuity of 6/36 on snellens vision chart with average CFT(central foveal thickness )of $563 \mu \mathrm{m}$. After 2 years the mean OCT measured CFT improved to $463 \mu \mathrm{m}$. In most of the patients vision is improved by 15 letters i.e. approximately 3 lines while few patients had stability of vision and very few no. of patients had deterioration of vision.

Conclusion: Ranibizumab is associated with clinically and statistically significant benefit with respect to visual acuity and reduction of CFT.
\end{abstract}

\section{Introduction}

Diabetic CSME, wet ARMD and retinal vein occlusion are leading cause of irreversible blindness in modern age era.

Diabetic macular oedema (DME) is a leading cause of vision impairment among people within the working age population. An estimated 346 million people are affected by diabetes worldwide in 2011 and the number of people with diabetes is expected to double from 2005 to 2030 and diabetic macular oedema is the most frequent cause of vision loss related to diabetes. The Wisconsin Epidemiologic study of Diabetic retinopathy found the 14 year incidence in DME in type 1 diabetics to be $26 \%$.Similarly the Diabetes Control and Complication Trial (DCTT) reported that $27 \%$ of type 1 diabetic patients develop DME within 9 years of onset .Development of DME occurs as a result of vascular endothelial damage with breakdown of blood retinal barrier. Hypoxia caused by microvascular disease stimulates the release of VEGF-A which is a major contributor to vascular permeability and angiogenesis.

Laser photocoagulation has been the mainstay of treatment for macular oedema since the publication of the results of Early Treatment Diabetic Retinopathy Study which showed an approx. 50\% reduction in the rate of moderate visual loss at 3 year following laser photocoagulation compared to no treatment along with it intensive blood pressure and blood sugar control have become the standard of care. Within the last 5 year intravitreal anti VEGF have come to common practice for management of DME and several recent randomized clinical trials have improved the effectiveness of Ranibizumab .

Age related macular degeneration(ARMD) has been recognized as one of the leading cause of vision loss in elderly people in western population. ARMD is a progressive disease of the central area In the posterior segment which leads to gradual deterioration of central vision .In the united States about 20-25\% of people of over80 years of age were estimated to have ARMD in 2000 and this number is expected to be more than 2.95 million in2020.People with ARMD have been found to experience reduced quality of life, depression and difficulty with activities of daily living.

There are two distinct form of ARMD: Dry or atrophic ARMD and wet or exudative ARMD. The exudative ARMD accounts for 10-20\% of total patients with macular degeneration and is associated with formation of neovascular membrane below the retina that release blood and fluid distorting central vision. Eventually these neovascular lesion result in scar leading to irreversible central vision loss.

Various studies shows the VEGF plays an important role in pathogenesis of this process(Aiello et al 1994;Kvanta et al 1996;Kliffen et al 1997;Boyd et al 2002).The observation that VEGF-A play a crucial part in angiogenesis leading to Neovascular ARMD has made a target of continuous investigation. Over the past 
decades there have been variety of medical therapies introduced with variable success to treat exudative form of ARMD in which anti VEGF is found to be superior in all.

Retinal vein occlusion (RVO) is a second most common cause of retinal vascular disease after diabetic retinopathy resulting in potentially irreversible loss of vision.RVO can be divided into two categories depending on the site of obstruction: central RVO (CRVO) when occlusion involved the whole central retinal vein and branch RVO(BRVO) when the occlusion involves only one branch of central retinal vein.BRVO occurs 2-3 times more commonly than CRVO.

Pathogenesis of RVO is multifactorial with thrombus formation being the primary cause but other possible causes are external compression of the central retinal vein .Main cause of vision loss in RVO is macular oedema. Until recently macular grid laser photocoagulation was treatment of macular oedema secondary to RVO .The humanised monoclonal antibody fragment ranibizumab bind to and inhibits vascular endothelial growth factor, which is a key driver of macular oedema in RVO.In 2010 ranibizumab was approved in the USA for treatment of macular oedema in RVO and in 2011 ranibizumab was approved in the European Union for treatment of visual impairment caused by macular oedema secondary to RVO .Anti VEGF therapies have been approved for ocular use for 10 years, initially for treatment of neovascular or wet ARMD later on it has been approved for treatment of Diabetic macular oedema and RVO Four inhibitors of VEGF-A are currently for clinical use(pegaptanib, bevacizumab, ranibizumab and aflibercept).Recent studies using anti VEGF inhibitors injected into vitreous cavity have shown promising result in these diseases.

Ranibizumab(lucentis, Genentech,South San Francisco)is a recombinant monoclonal antibody fragment directed towards all isoforms of VEGF-A that was specially designed to target Exudative ARMD.The human antibody fragment is produced by an E.Coli expression system and has a molecular weight of $48 \mathrm{kd}$ allowing for excellent retinal penetration.

\section{Material and method}

This study was conducted in Upgraded department of Ophthalmology N.S.C.B.Medical College Jabalpur in a duration of 2 years from Oct2014 -Sept2016.

100 eyes of 100 patients (48 females and 52males) with diabetic CSME (50 eyes),Wet ARMD(40eyes) and retinal vein occlusion(10 eyes) were enrolled in this study.

Following were exclusion criteria of patients-

1. Patients having known hypersensitivity to the active substance of Ranibizumab or to any of its excipients

2. Active or suspected ocular or periocular infection

3. Active severe intraocular inflammation

4. Advanced cataract

5. Uncontrolled blood sugar

6. Ischaemic central retinal vein occlusion/branch retinal vein occlusion

We took careful history of selected patients regarding nature and duration of symptoms and medical history. We did thorough clinical examinations include slit lamp biomicroscopy and measurement of visual acuity using snellens visual acuity chart as assessment of visual acuity is essential .Pre injection OCT(Spectral domain) evaluation was done and after each injection same was repeated again and at the completion of therapy . Each injection of Ranibizumab was given with the controlled blood sugar profile. Patients were given pre injection counselling regarding profile of therapy. Informed consent of all the patients enrolled in study was duly taken before intravitreal injection.

Methods of administration - The injection procedure carried out under aseptic conditions which includes the use of surgical hand disinfection, sterile gloves, sterile drape and a sterile eyelid speculum. Sterile paracentesis equipment was available as a precautionary measure.

Adequate anaesthesia and a broad spectrum topical microbiocide to disinfect the periocular skin, eyelid and ocular surface was administered prior to injection .Patients were intravitreally injected $0.3 \mathrm{mg}$ of Ranibizumab as $0.05 \mathrm{ml}$ under aseptic precaution on a monthly basis followed by treat and extend norms as and when required. Pre and post operatively patients were advised to instil topical antibiotic and topical antiglaucoma medication for 7 days along with systemic anhydrase inhibitor for 3 days. Indirect ophthalmoscopy was performed in each and every patient on day 1,3 and 7 and at completion of therapy i.e. After 2 year.OCT (Spectral domain)evaluation was done prior to injection as well as after every intravitreal injection and finally after completion of therapy. Similarly visual acuity was gauged in the same sitting. 


\section{Result}

Between 2014 -2016, 100 patients with diabetic CSME(50 eyes), neovascular ARMD (40 eyes) and retinal vein occlusion(10 eyes) were enrolled in this study. The mean age of these patients was 70 years. Prior to starting therapy following were the details-

- $\quad$ Patients of diabetic CSME had average CFT thickness of $563 \mu \mathrm{m}$ while most patients had visual acuity of 6/36(37 eyes)

- $\quad$ patients of wet ARMD had average CFT thickness of $563 \mu \mathrm{m}$ while most of the patients had average visual acuity of 6/60(27 eyes)

- patients of retinal vein occlusion had average CFT thickness of $672 \mu \mathrm{m}$ while most of the patients had visual acuity of $6 / 60(6$ eyes)

After 2 year of therapy following result occurred -

- Significant improvement in visual acuity was detected in most of the patients associated with a significant decrease in the central retinal thickness measured by OCT .The mean central retinal thickness decreased by $100 \mu \mathrm{m}$ and the mean visual acuity increased by 15 letters that is approximately 3 lines

- Few patients had stabilization of vision .

- Very few patients had deterioration of vision

Main causes were -

(a) Discontinuation of therapy by patients

(b) $50 \%$ of the lesion made up of serous pigment epithelial detachment.

(c) RPE tear

(d) Subretinal fibrosis

(e) Variability of patient's response to therapy

Chart no.1

\begin{tabular}{|c|c|c|}
\hline No. of Eyes & Male & Female \\
\hline 100 & $48(48 \%)$ & $52(52 \%)$ \\
\hline
\end{tabular}

Chart showing gender profile of patients

Chart no.2

\begin{tabular}{|c|c|c|}
\hline No. of Eyes & Average CFT Thickness & $\begin{array}{c}\text { Average Visual } \\
\text { Acuity }\end{array}$ \\
\hline 50 & $563 \mu \mathrm{m}$ & $6 / 60$ (10 eyes) \\
& & $6 / 36$ (37 eyes) \\
& & $6 / 24$ (03 eyes) \\
\hline
\end{tabular}

Chart showing profile of diabetic CSE before therapy

Chart no.3

\begin{tabular}{|c|c|c|}
\hline $\begin{array}{c}\text { No. of } \\
\text { Eyes }\end{array}$ & $\begin{array}{c}\text { Average CFT } \\
\text { Thickness }\end{array}$ & $\begin{array}{c}\text { Average Visual } \\
\text { Acuity }\end{array}$ \\
\hline 40 & $563 \mu \mathrm{m}$ & $6 / 60(27$ eyes) \\
& & $6 / 36$ (08 eyes) \\
& & $6 / 24$ (05 eyes) \\
\hline
\end{tabular}

Chart showing profile of wet ARMD before intravitreal Ranibizumab

Chart no.4

\begin{tabular}{|c|c|c|}
\hline No. of Eyes & Average CFT Thickness & Average Visual Acuity \\
\hline 10 & $672 \mu \mathrm{m}$ & $6 / 60$ (06 eyes) \\
& & $6 / 36(03$ eyes) \\
& & $6 / 24$ (01 eyes) \\
\hline
\end{tabular}


Chart showing profile of retinal vein occlusion before intravitreal Ranibizumab Follow up -

Chart no.5

\begin{tabular}{|l|c|c|}
\hline \multicolumn{1}{|c|}{ Improvement } & $\begin{array}{c}\text { No. of } \\
\text { Eyes }\end{array}$ & Percentage \\
\hline $\begin{array}{l}\text { 1. Reduction of CFT by } 100 \mu \mathrm{m} \\
\text { 2.Improvement of VA by 15 letters }\end{array}$ & 34 & $68 \%$ \\
\hline \multicolumn{1}{|c|}{ No Improvement } & $24 \%$ \\
\hline $\begin{array}{l}\text { 1.Stabilization of CFT } \\
\text { 2.Stabilization of VA }\end{array}$ & 12 & \\
\hline $\begin{array}{l}\text { Deterioration } \\
\text { 1.Inctease CFT by 100 } \mu \text { Detrioration } \\
\text { 2.Deterioration of VA by } 15 \text { letters }\end{array}$ & 04 & $8 \%$ \\
\hline
\end{tabular}

Chart showing profile of diabetic CSME after intravitreal Ranibizumab

Chart no.6

\begin{tabular}{|l|c|c|}
\hline \multicolumn{1}{|c|}{ Improvement } & $\begin{array}{c}\text { No. of } \\
\text { Eyes }\end{array}$ & Percentage \\
\hline $\begin{array}{l}\text { 1. Reduction of CFT by } 100 \mu \mathrm{m} \\
\text { 2. Improvement of VA by } 15 \text { letters }\end{array}$ & 24 & $60 \%$ \\
\hline \multicolumn{1}{|c|}{ No Improvement } \\
\hline $\begin{array}{l}\text { 1.Stabilization of CFT } \\
\text { 2.Stabilization of VA }\end{array}$ & 11 & $27.5 \%$ \\
\hline \multicolumn{1}{|c|}{ Deterioration } & & \\
\hline $\begin{array}{l}\text { 1. Increase CFT by } 100 \mu \mathrm{m} \\
\text { 2.Deterioration of VA }\end{array}$ & 05 & $12.5 \%$ \\
\hline
\end{tabular}

Chart showing profile of wet CSME patients after intravitreal injection of Ranibizumab

Chart no.7

\begin{tabular}{|l|c|c|}
\hline \multicolumn{1}{|c|}{ Improvement } & $\begin{array}{c}\text { No. of } \\
\text { Eyes }\end{array}$ & Percentage \\
\hline $\begin{array}{l}\text { 1.Reduction of CFT by 100 } \mu \mathrm{m} \\
\text { 2.Improvement of VA by 15 letters }\end{array}$ & 05 & $50 \%$ \\
\hline \multicolumn{2}{|c|}{ No Improvement } & $30 \%$ \\
\hline $\begin{array}{l}\text { 1.Stabilization of CFT } \\
\text { 2.Stabilization of VA }\end{array}$ & 03 & \\
\hline Deterioration & 02 & $20 \%$ \\
$\begin{array}{l}\text { 1.Increase CFT by 100 } \mu \mathrm{m} \text { Deterioration } \\
\text { 2.Deterioration of VA by 15 letters }\end{array}$ & 02 \\
\hline
\end{tabular}


Chart showing profile of retinal vein occlusion after intravitreal Ranibizumab

\section{Chart no.8}

Common Complication after Ranibizumab injection

\begin{tabular}{|l|c|c|}
\hline \multicolumn{1}{|c|}{ Complications } & Eyes & Percentage \\
\hline 1. Pain at the site of injection & 90 & $90 \%$ \\
\hline 2. Multiple or single bubble & 84 & $84 \%$ \\
\hline 3. Endophthalmitis & 01 & $1 \%$ \\
\hline 4. Non fatal stroke(CVA) & 02 & $2 \%$ \\
\hline
\end{tabular}

\section{Discussion}

The present study comprises of 100 patients who took treatment of diabetic CSME, wet ARMD and retinal vein occlusion with Ranibizumab. The patients got a total mean of 5.6 injections during 2 year period, 3 injections during upload phase and 1.5 injections during the maintenance phase of 12 months. Followed by treat and extend norm. After 2 years of follow up most of the patients had improvement of visual acuity as well as reduction of CFT(diabetic CSME-68\%, wet CSME-60\%,RVO-50\%)Few patients had no improvement (diabetic CSME-24\%,wet ARMD-27.5\%,RVO-30\%)and very small no. of patients had deterioration i.e. increased CFT and diminished visual acuity (diabetic CSME-8\%,wet ARMD-12.5\%RVO-20\%).

Diminished visual acuity occurs mostly due to discontinuation of therapy,various coexistent retinal pathology like RPE tear,subretinal Fibrosis ,subretinal fluid collection and variability of response of intravitreal Ranibizumab in different patients.

As Diabetic CSME,ARMD and RVO are complex diseases and there are a number of other factors that could impact response to anti VEGF therapy.Although generally elevated in patients of Diabetic CSME,ARMD and RVO ,levels of VEGF have been observed to be highly variable in the vitreous of different patients.Furthermore there is an upregulation of a multitude of growth factors and cytokines that contribute to the breakdown of blood retinal barrier and consequent vascular leakage responsible for macular oedema including angiopoietins, tumor necrosis factors, interleukins and matrix metalloproteinases.Therefore it is unsurprising that a variability in patients response to anti VEGF therapy has been observed in several studies. Our study emphasized role of Ranibizumab in wet ARMD,Diabetic CSME and RVO as not only prevention of vision loss but also mean improvement of vision.

\section{Conclusion}

Our study showed Ranibizumab was associated with clinically and statistically significant benefits with respect to visual acuity and CFT during 2 years of follow up. Visual acuity was improved and central retinal thickness decreased in patients with diabetic CSME, wet ARMD and retinal vein occlusion after Ranibizumab in an appreciable way. In this way it is an excellent drug for management of these diseases with minimal side effects.

\section{References}

[1]. Baseline and Early Natural History Report .The Central Vein Occlusion Study.Arch Ophthalmol1993;111:1087-95

[2]. Aiello LP,Avery RL ,Arrigg PG et al.Vascular endothelial growth factor in ocular fluid of patients with diabetic maculopathy nd other retinal disorder .N Eng J Med 1994;331:1480-7

[3]. Rosenfeld PJ,Brown DM,Heier JS,Boyer DS,KaiserPK,Chung CY et al.Ranibizumab for neovascular age related macular degeneration. $N$ Engl J Med 2006;355(14):1419-1431

[4]. Wong TY,Liew G,Mitchel P.Clinical update : new treatments for age related macular degeneration.Lacet.2007;370

[5]. Fung,AE,Lalwani,GA,Rosenfeld,PJ,et al An optical coherence tomography guided, variable-dosing regimen with intravitreal ranibizumab(Lucentis)for neovascular age related macular degeneration.Am J Ophthalmol2007;143:566583

[6]. Pieramici DJ,Rabena M,Castellarin AA,Nasir M,See R,Norton et al .Ranibizumab for thetreatment of macular edema associated with perfused central retinal vein occlusions.Ophthalmology 2008;115(10):e47-e54

[7]. Genentech.FDA Approves Lucentis (Ranibizumab Injection) for treatment of Macular Oedema Following Retinal Vein Occlusion.2010 
[8]. Nguyen QD ,ShahSM,Khwaja AA,Channa R,Hatef E,Do DV,Boyer D,Heier JS,Abraham P,ThachABet al.Two year outcomes of the Ranibizumab for edema of the macula in daibetes(READ- 2)study.Ophthalmology.2010;117:21462151

[9]. Novartis .Novartis gains new indications for lucentis in EU for vision loss due to Diabetic Macular Edema,a leading cuse of blindness.2011

[10]. Nguyen QD,Brown DM,Marcus DM,Boyer DS,Patel S,Feiner L,et al.Ranibizumab for diabetic macular edema:results from 2 phase III randomized trials:RISE and RDE.Ophthalmology. 2012;119:789-801

[11]. Lattanzio R,Torres Gimeno A,Battaglia Parodi M,Bandello F.Retinal vein occlusion:current treatment.Ophthalmologica2011;225(3):135-143

[12]. Elman MJ,Aiello P,Beck Rwet al.Diabetic Retinopathy Clinical research network.Randomized trial evaluating ranibizumab plus prompt or deferred laser or triamcinolone plus prompt laser for diabetic macular oedema.Ophthalmology 2010;117:1064-77 